\section{REFERENCES}

1.Fajkus, J., M. Simickova, and J. Malaska. 2002. Tiptoeing to chromosome tips: facts, promises and perils of today's human telomere biology. Philos. Trans. R. Soc. Lond. B Biol. Sci. 357:545-562.

2.Kim, N.W., M.A. Piatyszek, K.R. Prowse, C.B. Harley, M.D. West, P.L. Ho, G.M. Coviello, W.E. Wright, et al. 1994. Specific association of human telomerase activity with immortal cells and cancer. Science 266:2011-2015.

3.Wege, H., M.S. Chui, H.T. Le, J.M. Tran, and M.A. Zern. 2003. SYBR Green real-time telomeric repeat amplification protocol for the rapid quantification of telomerase activity. $\mathrm{Nu}$ cleic Acids Res. 31:E3-3.

4.Wu, K.D., L.M. Orme, J. Shaughnessy, Jr., J. Jacobson, B. Barlogie, and M.A.S. Moore. 2003. Telomerase and telomere length in multiple myeloma: correlations with disease heterogeneity, cytogenetic status, and overall survival. Blood 101:4982-4989.

Received 31 July 2003; accepted 11 September 2003.

Address correspondence to Jiri Fajkus, Institute of Biophysics, Academy of Sciences of the Czech Republic, Královopolská 135, CZ-61265 Brno,Czech Republic.e-mail:fajkus@ibp.cz.

\section{Blocking oligonucleotides improve sequencing through inverted repeats}

\author{
Dominic Esposito, William K. Gillette, and James L. Hartley \\ National Cancer Institute, Frederick, MD, USA
}

BioTechniques 35:914-920 (November 2003)
Gateway ${ }^{\mathrm{TM}}$ cloning is a popular method for genome analysis and protein expression (for a compilation of publications using Gateway, see http:// www.ncifcrf.gov/rtp/pel/pubs.asp). In Gateway cloning, bacteriophage $\lambda$ recombination sites, called att sites, are first added to the DNA sequence of interest, typically during PCR. The presence of these flanking att sites allows the DNA of interest to be cloned and subsequently subcloned into expression vectors entirely by in vitro site-specific recombination reactions (1). The recombination reactions are efficient compared to those based on ligases, and powerful

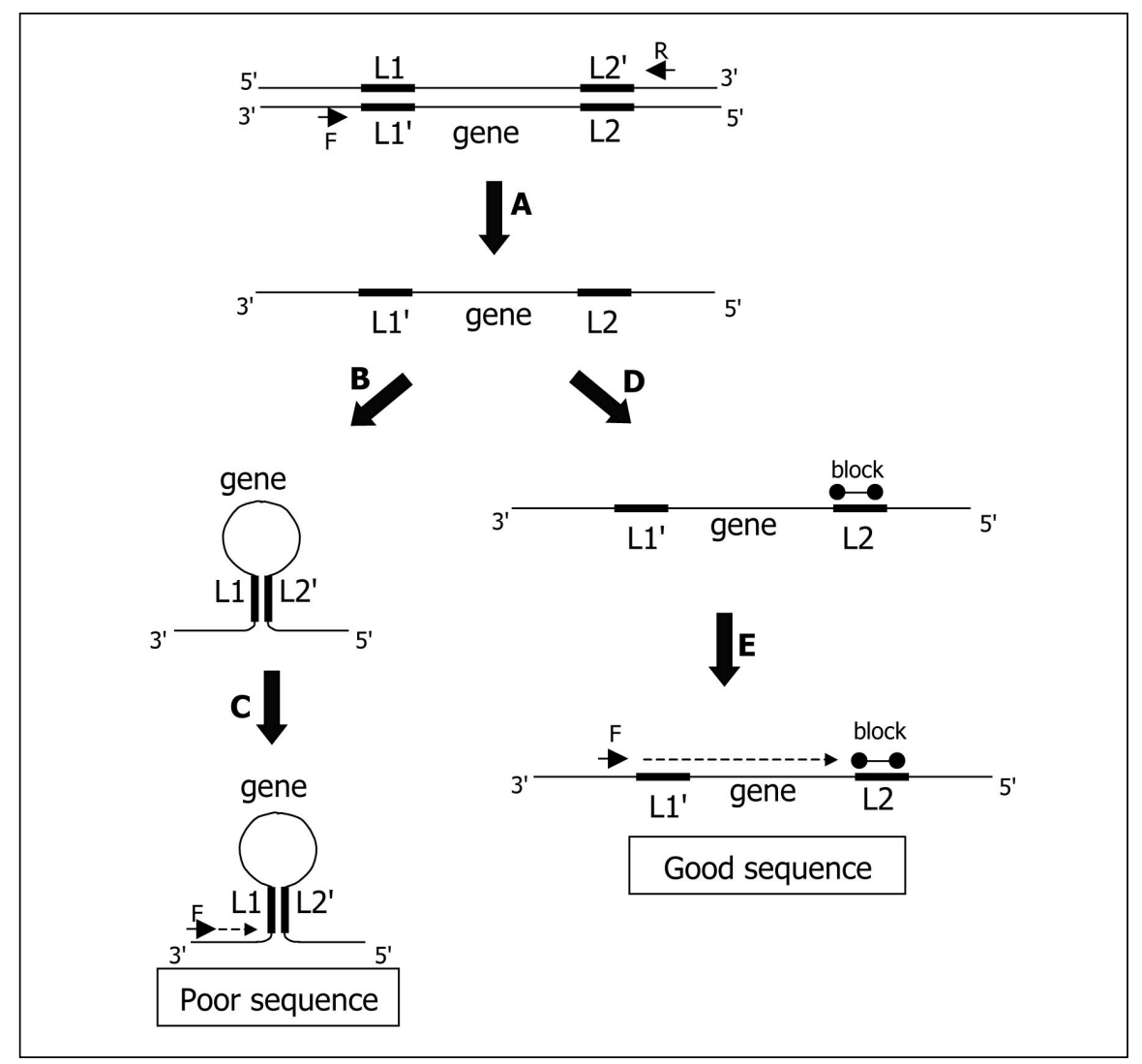

Figure 1. Sequencing through inverted repeats. Gateway entry clones contain the gene (or DNA of interest) between inverted repeats of 100-bp at $\mathrm{L}$ sites ( $95 \mathrm{bp}$ inverted repeats, 3 mismatches). (A) During sequencing, the duplex DNA is denatured to single strands (bottom strand shown from $3^{\prime}$ to $5^{\prime}$ ). (B) As the size of the gene decreases to less than $1 \mathrm{~kb}$, the inverted repeats on each strand pair to each other more efficiently. (C) Subsequent annealing of the sequencing oligonucleotide ( $F$, forward) results in the extension of the sequencing reaction being stopped at the double-stranded region of the hairpin. (D) Formation of the hairpin can be inhibited by the addition of a blocking oligonucleotide that is complementary to the distal repeat att $\mathrm{L} 2$, which allows (E) the annealing and extension of the sequencing primer to proceed. Note that because $a t t \mathrm{~L} 1$ and $a t t \mathrm{~L} 2$ are almost identical, the blocking oligonucleotide also anneals to att $\mathrm{L} 1$, thus the same blocking oligonucleotide facilitates sequencing from both forward and reverse primers. 
Table 1. Sequences of att L1 and $a t t \mathrm{~L} 2$ in Entry Clones Derived from Recombination Between pDonr223 and PCR Products Containing attB1.1 and 2.1

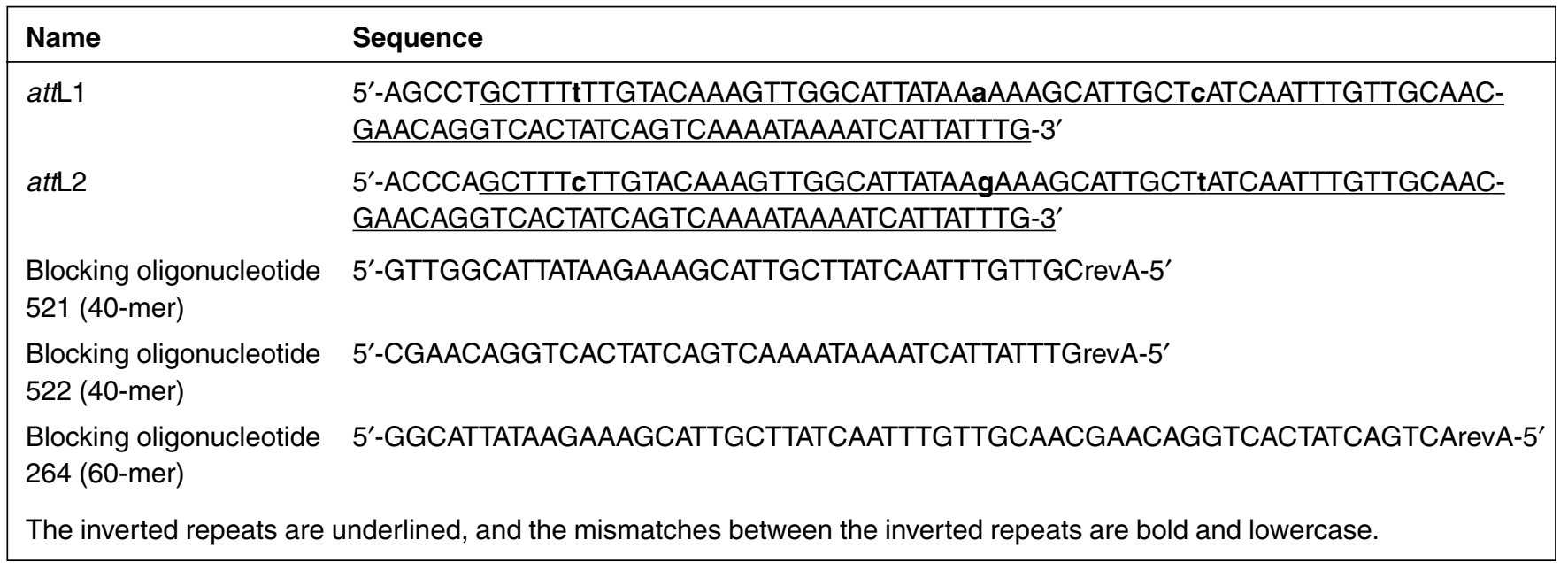

selections and uniform conditions allow for highly efficient and automated cloning. For example, 12,000 predicted open reading frames of Caenorhabditis elegans have been amplified, and the re- sulting PCR products have been cloned by this method (2).

Gateway clones of PCR products are called entry clones, and the inserts in these clones are typically sequenced

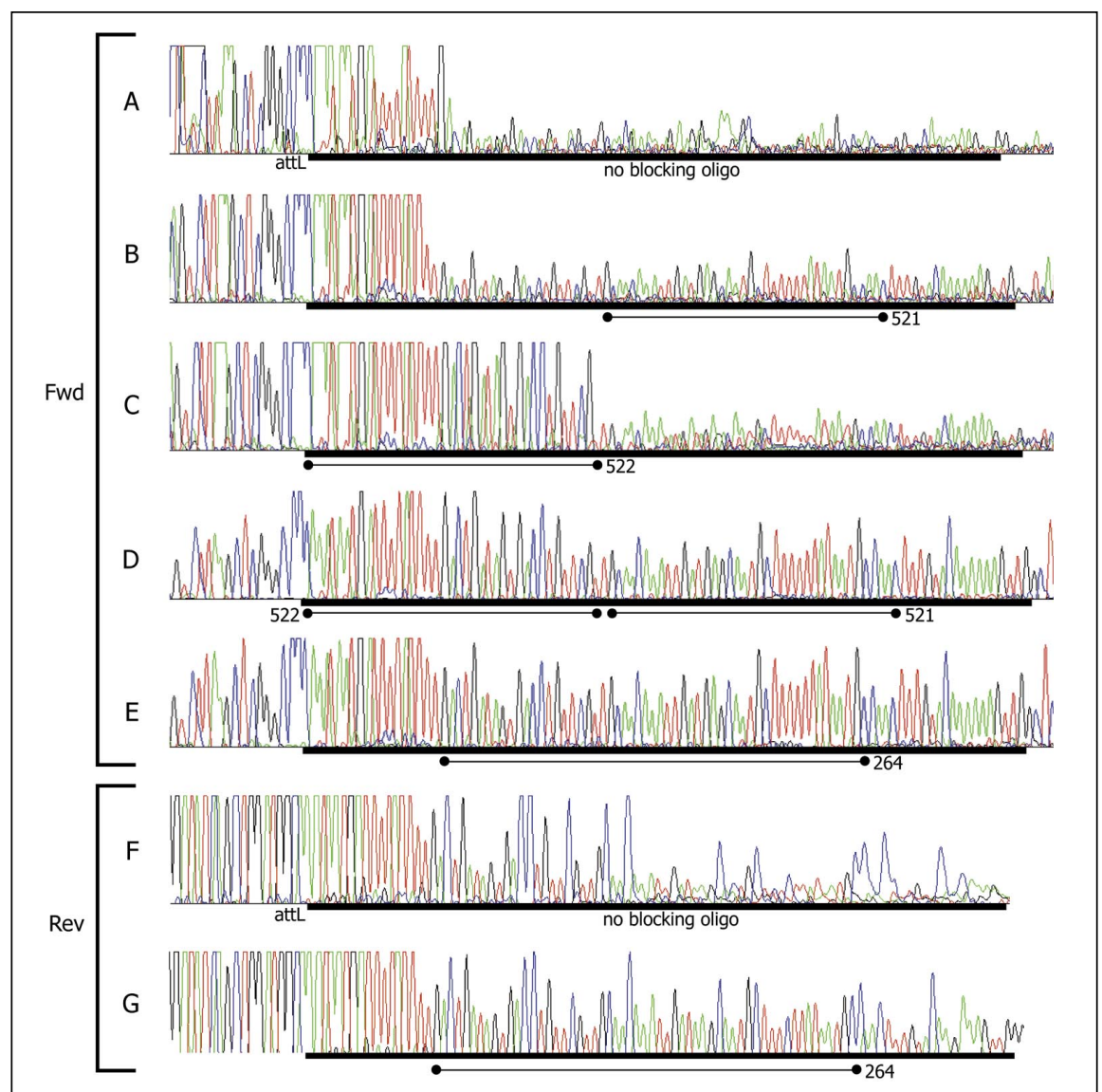

Figure 2. Improved sequencing of a 390-bp insert entry clone by the addition of blocking oligonucleotides. Scans A-E were obtained using the forward sequencing primer, and scans F-G were obtained using the reverse sequencing primer. The black bar shows the locations of (scans A-E) att $\mathrm{L} 1$ or (scans $\mathrm{F}-\mathrm{G}$ ) att $\mathrm{L} 2$. The blocking oligonucleotides hydridize to the distal $a t t \mathrm{~L}$ site on each strand (attL2 on the bottom strand using the forward sequencing primer, and $a t \mathrm{~L} 1$ on the top strand using the reverse sequencing primer). Fwd, forward; Rev, reverse.

to detect and discard plasmids that have mutations that resulted from primer synthesis or PCR errors. In the process of sequencing several hundred inserts in entry clones, we have noticed that sequence read lengths drop as the sizes of the genes decrease. With inserts smaller than approximately $500 \mathrm{bp}$, the quality of sequence data obtained under standard sequencing conditions is poor. This is presumably due to the presence of inverted repeats of 100-bp att $\mathrm{L}$ recombination sites that flank DNA segments in entry clones. Because each sequencing cycle produces single-stranded template DNA, the nearly identical $a t t$ Ls can hybridize to form a 15-bp hairpin. If the hairpin forms before the sequencing primer can be extended through the att $\mathrm{L}$, then sequence is not obtained for that cycle (Figure 1). Here we describe a simple way to minimize this difficulty.

pDonr223 is identical to pDonr221 (Invitrogen, Carlsbad, CA, USA), except that it confers spectinomycin resistance instead of kanamycin resistance. Blocking, sequencing, and PCR oligonucleotides were purchased from Qiagen (Valencia, CA, USA) and desalted but not otherwise purified. The blocking oligonucleotides contained their $3^{\prime}$-terminal base in reverse orientation $\left(3^{\prime} \rightarrow 3^{\prime}\right.$ linkage) so that both ends of the blocking oligonucleotides were $5^{\prime} \mathrm{OH}$. Modified $a t t \mathrm{~B}$ sequences were used for improved cloning efficiency (3): attB1.1 was added to $5^{\prime}$ primers $5^{\prime}$-GGGGACAACTTTGTACAAAAAAGTTG-3', and $a t t$ B2.1 was added to $3^{\prime}$ primers $5^{\prime}$-GGGGACAACTTTGTACAAGAAAGTTG-3'. 
Table 2. Effect of Blocking Oligonucleotide 264 (60-mer) on Phred Scores, Forward and Reverse Primers, and with Clones of Various Sizes

\begin{tabular}{|c|c|c|c|}
\hline $\begin{array}{l}\text { Insert } \\
\text { Size } \\
\text { (bp) }\end{array}$ & $\begin{array}{l}\text { Sequencing } \\
\text { Primer }\end{array}$ & $\begin{array}{c}\text { Blocking } \\
\text { Oligonucleotide } \\
264 \\
\text { Concentration } \\
(\mu \mathrm{M})\end{array}$ & $\begin{array}{c}\text { Phred } \\
\text { Scores }^{a}\end{array}$ \\
\hline \multirow[t]{2}{*}{390} & Forward & $\begin{array}{r}0 \\
1 \\
5 \\
10 \\
20\end{array}$ & $\begin{array}{r}5 \\
201 \\
316 \\
351 \\
484\end{array}$ \\
\hline & Reverse & $\begin{array}{r}0 \\
25\end{array}$ & $\begin{array}{l}152 \\
460\end{array}$ \\
\hline \multirow[t]{2}{*}{425} & Forward & $\begin{array}{r}0 \\
25\end{array}$ & $\begin{array}{r}69 \\
378\end{array}$ \\
\hline & Reverse & $\begin{array}{r}0 \\
25\end{array}$ & $\begin{array}{r}94 \\
458\end{array}$ \\
\hline \multirow[t]{2}{*}{600} & Forward & $\begin{array}{r}0 \\
5 \\
10 \\
25 \\
50\end{array}$ & $\begin{array}{l}120 \\
404 \\
630 \\
663 \\
664\end{array}$ \\
\hline & Reverse & $\begin{array}{r}0 \\
5 \\
10 \\
25 \\
50\end{array}$ & $\begin{array}{r}0 \\
89 \\
152 \\
155 \\
460\end{array}$ \\
\hline \multirow[t]{2}{*}{638} & Forward & $\begin{array}{r}0 \\
25\end{array}$ & $\begin{array}{r}66 \\
656\end{array}$ \\
\hline & Reverse & $\begin{array}{r}0 \\
25\end{array}$ & $\begin{array}{l}237 \\
587\end{array}$ \\
\hline \multirow[t]{2}{*}{719} & Forward & $\begin{array}{r}0 \\
\text { n.d. }\end{array}$ & $\begin{array}{l}319 \\
\text { n.d. }\end{array}$ \\
\hline & Reverse & $\begin{array}{r}0 \\
25\end{array}$ & $\begin{array}{l}167 \\
518\end{array}$ \\
\hline \multirow[t]{2}{*}{887} & Forward & $\begin{array}{r}0 \\
25\end{array}$ & $\begin{array}{l}266 \\
642\end{array}$ \\
\hline & Reverse & $\begin{array}{r}0 \\
\text { n.d. }\end{array}$ & $\begin{array}{r}56 \\
\text { n.d. }\end{array}$ \\
\hline $\begin{array}{l}\text { n.d., nc } \\
\text { aseque }\end{array}$ & $\begin{array}{l}\text { determined } \\
\text { nce read lengt }\end{array}$ & & \\
\hline
\end{tabular}

frame (1). PCR was performed by standard techniques. Amplification products were purified with QIAquick $^{\mathrm{TM}}$ (Qiagen) PCR kits and then cloned into pDonr223 with Gateway BP Clonase ${ }^{\mathrm{TM}}$, according to the manufacturer's instructions (Invitrogen). Escherichia coli (DH5 $\alpha)$ colonies were cultured overnight in CIRCLEGROW $^{\circledR} \quad$ (Qbiogene, Carlsbad, CA, USA), and sequencing template plasmid was purified from $1 \mathrm{~mL}$ of cells by manual alkaline sodium dodecyl sulfate (SDS) extraction and dissolved to 150-200 $\mathrm{ng} / \mu \mathrm{L}$ in TE $(10 \mathrm{mM}$ Tris-HCl, $\mathrm{pH}$ 8.0, 0.1 mM EDTA) containing $50 \mu \mathrm{g} / \mathrm{mL}$ RNase A. Sequencing reactions comprising $6 \mu \mathrm{L}$ miniprep plasmid DNA, 3.2 pmol sequencing primer, and $1 \mu \mathrm{L}$ blocking oligonucleotide (for concentrations, see below) were brought to a final volume of 14 $\mu$ L. An aliquot (3.85 $\mu \mathrm{L})$ of this mixture was combined with $2.2 \mu \mathrm{L}$ ABI PRISM ${ }^{\circledR}$ BigDye $^{\text {TM }}$ terminators (version 1; Applied Biosystems, Foster City, CA, USA), $1.1 \mu \mathrm{L}$ BigDye v1.1 $5 \times$ dilution buffer (Applied Biosystems), and $3.85 \mu \mathrm{L}$ water, cycled Sequencing primers were forward, 5' - CCCAGTCACGACGTTGTAAAACG-3', and reverse, 5'-GTAACATCAGAGATTTTGAGACAC-3'. Table 1 shows the sequences of $a t t \mathrm{~L} 1$ and $a t t \mathrm{~L} 2$ in entry clones derived from recombination between pDonr223 and PCR products containing attB1.1 and 2.1.

The $a t t \mathrm{~B} 1.1$ and $a t t \mathrm{~B} 2.1$ sequences were added to the $5^{\prime}$ ends of amplification primers in the appropriate reading
25 times at $96^{\circ}$ for $10 \mathrm{~s}, 50^{\circ}$ for $5 \mathrm{~s}$, and $60^{\circ} \mathrm{C}$ for $4 \mathrm{~min}$, and applied to an $\mathrm{ABI}$ PRISM 3700 sequencer (Applied Biosystems). Note that higher concentrations of blocking oligonucleotides might be required when using more dilute BigDye terminator mixture.

Figure 1 shows the mechanism by which the inverted att $\mathrm{L}$ sites of Gateway entry clones are presumed to inhibit the extension of sequencing primers. As the size of the cloned DNA segment decreases, the rate at which the complementary att $\mathrm{L}$ sites find each other and hybridize to form a hairpin increases. Because each sequencing cycle begins with a denaturation step that produces a single-stranded template, if the hairpin forms before the sequencing primer is extended through the proximal at $\mathrm{L}$, then the extension of the sequencing primer in each cycle will be impeded.

We expected to diminish this undesirable process by making the distal att $\mathrm{L}$ site double-stranded with complementary oligonucleotides before it could hybridize to the proximal att $\mathrm{L}$ site. We did this by adding oligonucleotides that were blocked at their $3^{\prime}$ termini and complementary to the distal att $\mathrm{L}$ site to each sequencing reaction. We tested several candidate oligonucleotides for their effect on the sequencing reactions of a template with an insert of $390 \mathrm{bp}$ cloned between the $a t t \mathrm{~L} 1$ and $a t t \mathrm{~L} 2$ inverted repeats (Figure 2). Without any blocking oligonucleotide (Figure 2A), the extension of the forward primer was stopped approximately 20 bases into the stem of the hairpin, which is AT-rich. The addition of blocking oligonucleotide 521 (Figure 2B), which hybridizes toward the top of the stem of the hairpin, actually decreased the read length to 17 bases into the stem. Oligonucleotide 522, which hybridizes at the bottom of the stem, gave very good sequence over its length of 39 bases (Figure 2C), at which point the signal strength dropped dramatically. The addition of both 521 and 522 (Figure 2D) allowed for sequence to be read through the hairpin, but better signal strength was seen with oligonucleotide 264 , which is a single, longer oligonucleotide (Figure 2E) (60-mer).

The sequence quality with the reverse primer was not as severely affected by the hairpin formation (Figure $2 \mathrm{~F}$ ), and the sequence quality did not improve as dramatically with blocking oligonucleotide 264 (Figure 2G). Table 2 summarizes the results of several experiments with blocking oligonucleotide 264 at several oligonucleotide concentrations, forward and reverse sequencing primers, and cloned genes of various sizes. We then added 1 $\mu \mathrm{L} 25 \mu \mathrm{M}$ blocking oligonucleotide 264 to our entry clone sequencing reactions.

Inverted repeats represent difficult 
regions in genome sequencing projects (4-6). The method described here has limited applicability to these situations because one must know the sequence of the inverted repeats to design a blocking oligonucleotide. In such circumstances, it would be preferable to separate the inverted repeats by cloning them separately or to increase the distance between the inverted repeats by inserting a transposon (of known sequence) into the loop of the hairpin (7). Alternatively, one could design sequencing oligonucleotides with higher melting temperatures and attempt to minimize hairpin formation by raising the annealing temperature in each sequencing cycle. For sequencing Gateway entry clones using standard forward and reverse primers, the blocking oligonucleotide method described here is a less expensive and easier way to acquire long sequence reads.

\section{ACKNOWLEDGMENTS}

We thank Lynn Rasmussen and Claudia Stewart of the Laboratory of Molecular Technology, SAIC Frederick, for sequencing support. This project has been funded in whole or in part with Federal funds from the National Cancer Institute, National Institutes of Health, under grant no. N01-CO12400 (J.L.H.). The content of this publication does not necessarily reflect the views or policies of the Department of Health and Human Services, nor does the mention of trade names, commercial products, or organizations imply endorsement by the U.S. Government.

\section{REFERENCES}

1.Hartley, J.L., G.F. Temple, and M.A. Brasch. 2000. DNA cloning using in vitro site-specific recombination. Genome Res. 10:1788-1795.

2.Reboul, J., P. Vaglio, J.F. Rual, P. Lamesch, M. Martinez, C.M. Armstrong, S. Li, L. Jacotot, et al. 2003. C. elegans ORFeome version 1.1: experimental verification of the genome annotation and resource for proteome-scale protein expression. Nat. Genet. 34:35-41.

3.Cheo, D., M.A. Brasch, G.F. Temple, J.L. Hartley, and D. Byrd. 2001. Use of multiple recombination sites with unique specificity in recombinational cloning. International Patent application no. WO 01/42509 A1.

4.Ishikawa, T., Y. Hayashida, K. Hirayasu, K. Ozawa, N. Yamamoto, T. Tanaka, and S. Matsuura. 2003. Use of transcriptional sequencing in difficult to read areas of the genome. Anal. Biochem. 316:202-207.

5.Haqqi, X. Zhao, A. Panciu, and S.P. Yadav. 2002. Sequencing in the presence of betaine: improvement in sequencing of the localized repeat sequence regions. J. Biomol. Tech. 13: 265-271.

6.Flint, J., M. Sims, K. Clark, R. Staden, and K. Thomas. 1998. An oligo-screening strategy to fill gaps found during shotgun sequencing projects. DNA Seq. 8:241-245.

7.Davies, C.J. and C.A. Hutchison, III. 1991. A directed DNA sequencing strategy based upon Tn3 transposon mutagenesis: application to the $A D E 1$ locus on Saccharomyces cerevisiae chromosome I. Nucleic Acids Res.
19:5731-5738.

8.Ewing, B., L. Hillier, M.C. Wendl, and P. Green. 1998. Base-calling of automated sequencer traces using phred. I. Accuracy assessment. Genome Res. 8:175-185.

Received 18 June 2003; accepted 23 July 2003.

Address correspondence to Dominic Esposito, Protein Expression Laboratory, Building 327, SAIC/NCI, P.O. Box B, Frederick, MD 21702,USA.e-mail: domespo@ncifcrf.gov.

\title{
DNase I treatment of cDNA first strands prevents RT-PCR amplification of contaminating DNA sequences
}

\author{
Aljoscha M. Flohr ${ }^{1}$, Thomas Hackenbeck ${ }^{1}$, Claudia Schlueter $^{1}$, \\ Piere Rogalla ${ }^{2}$, and Joern Bullerdiek ${ }^{1}$ \\ ${ }^{1}$ Center for Human Genetics, University of Bremen and ${ }^{2}$ alcedo biotech, \\ Bremen, Germany
}

BioTechniques 35:920-926 (November 2003)

Retropseudogenes arise in evolution by reverse transcription of processed mRNAs and incorporation of the resulting cDNAs back into the genome (1). They are intronless DNA sequences that share a high degree of homology with the cDNA of their corresponding active genes. The human genome is estimated to contain approximately 23,000 to 33,000 retropseudogenes (2). An example of a gene family exhibiting retropseudogenes is the high mobility group protein family of which the HMGAl (HMGA protein family) and $H M G B 1$ (HMGB protein family) genes have been shown to contain many retropseudogenes (3-5). Several retropseudogenes have also been described for the housekeeping genes GAPDH and $\beta$-actin $(A C T B)(6,7)$. In a lot of cases, the sequence homology of the retropseudogenes compared with the functional mRNA can be so high that retropseudogenes may serve as an appropriate template in a PCR using genomic DNA and a primer set detecting a cDNA sequence of the active gene $(4,8)$.
Unfortunately, PCR products amplified from retropseudogenes cannot be distinguished in size from those amplified from cDNA, as retropseudogenes lack introns. A similar problem exists for specific amplification of intronless genes because in a RT-PCR, discrimination of cDNA and DNA amplification products by differing sizes of PCR products is not possible. Although the proportion of intronless human genes is generally thought to be no higher than $5 \%$ of all genes, there do exist some families of intronless genes like the histone genes and the G-protein-coupled receptor (GPCR) gene family (9).

Thus, genomic DNA (gDNA) contamination of cDNAs affects specific amplification of both housekeeping or target genes exhibiting at least one retropseudogene and intronless genes. Accordingly, a gDNA-free cDNA is needed to perform reliable RT-PCR analyses. Herein we describe an improvement of the standard method to eliminate detectable gDNA contamination in cDNA preparations. 